\title{
Physics
}

ISSN (Online): 1776-0852 ISSN (Print): 1402-9251

Journal Home Page: https://www.atlantis-press.com/journals/inmp

\section{A new solvable many-body problem of goldfish type}

Oksana Bihun, Francesco Calogero

To cite this article: Oksana Bihun, Francesco Calogero (2016) A new solvable many-body problem of goldfish type, Journal of Nonlinear Mathematical Physics 23:1, 28-46, DOI: https://doi.org/10.1080/14029251.2016.1135638

To link to this article: https://doi.org/10.1080/14029251.2016.1135638

Published online: 04 January 2021 


\title{
A new solvable many-body problem of goldfish type
}

\author{
Oksana Bihun \\ Department of Mathematics, University of Colorado, Colorado Springs, \\ 1420 Austin Bluffs Pkwy, \\ Colorado Springs, CO 80918, USA \\ obihun@uccs.edu \\ Francesco Calogero \\ Physics Department, University of Rome "La Sapienza" \\ p. Aldo Moro, I-00185 Roma, Italy \\ Istituto Nazionale di Fisica Nucleare, Sezione di Roma, Italy \\ francesco.calogero@romal.infn.it,francesco.calogero@uniroma1.it
}

Received 13 July 2015

Accepted 30 September 2015

\begin{abstract}
A new solvable many-body problem of goldfish type is introduced and the behavior of its solutions is tersely discussed.

Keywords: Solvable $N$-body problems; many-body problems; isochronous systems; completely periodic solutions; goldfish type systems.
\end{abstract}

2000 Mathematics Subject Classification: 70F10, 70K42.

\section{Introduction}

Notation 1.1. Hereafter $N$ is (unless otherwise indicated) an arbitrary integer, $N \geq 2$, the (generally complex) numbers $z_{n} \equiv z_{n}(t)$ are the dependent variables, $t$ ("time") is the independent variable, superimposed dots denote time-differentiations, and indices such as $n, m, \ell$ run over the integers from 1 to $N$ unless otherwise indicated (see for instance below in (1.1) the limitation $\ell \neq n$ on the $N$ values of $\ell$ ). Below we often omit to indicate explicitly the $t$-dependence of various quantities, when this can be done without causing misunderstandings. Hereafter $N \times N$ matrices are denoted by upper-case boldface letters (so that, for instance, the matrix $\mathbf{C}$ has the $N^{2}$ elements $C_{n m}$ ). Lowercase boldface letters stand for $N$-vectors (so that, for instance, the $N$-vector $\mathbf{z}$ has the $N$ components $z_{n}$ ); and the imaginary unit is denoted by $\mathbf{i}$ (so that $\mathbf{i}^{2}=-1$, and $\mathbf{i}$ is of course $n o t$ a $N$-vector!). We occasionally use the Kronecker symbol, with its standard definition: $\delta_{m n}=1$ for $m=n, \delta_{m n}=0$ for $m \neq n$. And let us mention the standard convention according to which an empty sum vanishes and an empty product equals unity, i. e. $\sum_{j=J}^{K}\left(f_{j}\right)=0, \prod_{j=J}^{K}\left(f_{j}\right)=1$ if $K<J$.

The prototypical "goldfish" many-body model [2] is characterized by the translation-invariant equations of motion

$$
\ddot{z}_{n}=\mathbf{i} \omega \dot{z}_{n}+\sum_{\ell=1, \ell \neq n}^{N}\left(\frac{2 \dot{z}_{n} \dot{z}_{\ell}}{z_{n}-z_{\ell}}\right)
$$


A Hamiltonian producing these equations of motion reads as follows:

$$
H(\zeta ; \mathbf{z})=\sum_{n=1}^{N}\left[\mathbf{i} \omega z_{n}+\exp \left(\zeta_{n}\right) \prod_{\ell=1, \ell \neq n}^{N}\left(z_{n}-z_{\ell}\right)^{-1}\right],
$$

where of course the $N$ coordinates $\zeta_{n} \equiv \zeta_{n}(t)$ are the canonical momenta corresponding to the canonical particle coordinates $z_{n} \equiv z_{n}(t)$. The solution of the corresponding initial-values problem is provided by the $N$ roots $z_{n} \equiv z_{n}(t)$ of the following, rather neat, algebraic equation in the variable $z$ :

$$
\sum_{\ell=1, \ell \neq n}^{N}\left[\frac{\dot{z}_{\ell}(0)+\mathbf{i} \omega z_{\ell}(0)}{z-z_{\ell}(0)}\right]=\frac{\mathbf{i} \omega}{\exp (\mathbf{i} \omega t)-1} .
$$

(Note that this is actually a polynomial equation of degree $N$ in $z$, as seen by multiplying it by $\prod_{m=1}^{N}\left[z-z_{m}(0)\right]$ ). Hence this model is isochronous (whenever the parameter $\omega$ is positive, as we generally assume hereafter; the special case $\omega=0$ is "the" prototypical, nonisochronous, case...): all its solutions are completely periodic, with the period $T=2 \pi / \omega$ or, possibly, due to an exchange of the particle positions through the motion, with a period that is a (generally small with respect to its possible maximal value $N$ !, see [6]) integer multiple of $T$.

Several solvable generalizations of the goldfish model, characterized by Newtonian equations of motion featuring additional forces besides those appearing in the right-hand side of (1.1a), are known: see for instance $[1,3-5]$ and references therein.

Remark 1.1. Above and hereafter we call a many-body model solvable if its initial value problem can be solved by algebraic operations, such as finding the $N$ zeros of a known $t$-dependent polynomial of degree $N$ (of course such an algebraic equation can be explicitly solved only for $N \leq 4$ ).

Recently a simple technique has been introduced [7], which allows to identify and investigate additional solvable models of goldfish type; and a few examples of such models yielded by this new approach have been identified and tersely discussed [7]. The model treated in this paper is another such new model, which is perhaps itself interesting (as all solvable models tend to be), and moreover is related to the properties of the zeros of (monic) polynomials of degree $N$ the coefficients of which are the zeros of Hermite polynomials (see, for instance, [8]) of degree $N$.

\section{The Model and its Solutions}

The Newtonian equations of motion of the new many-body problem of goldfish type read as follows:

$$
\begin{aligned}
& \ddot{z}_{n}=\sum_{\ell=1, \ell \neq n}^{N}\left(\frac{2 \dot{z}_{n} \dot{z}_{\ell}}{z_{n}-z_{\ell}}\right)-\left[\prod_{\ell=1, \ell \neq n}^{N}\left(z_{n}-z_{\ell}\right)\right]^{-1} . \\
& \cdot \sum_{m=1}^{N}\left\{\left(z_{n}\right)^{N-m}\left[-\omega^{2} c_{m}+2 \sum_{\ell=1, \ell \neq m}^{N}\left(c_{m}-c_{\ell}\right)^{-3}\right]\right\},
\end{aligned}
$$

with

$$
c_{m}=(-1)^{m} \sum_{1 \leq s_{1}<s_{2} \ldots<s_{m} \leq N}\left[\prod_{r=1}^{m}\left(z_{s_{r}}\right)\right] .
$$


Here and hereafter the symbol $\sum_{1 \leq s_{1}<s_{2} \ldots<s_{m} \leq N}$ signifies the sum from 1 to $N$ over the $m$ (integer) indices $s_{j}$ with $j=1, \ldots, m$ and the restriction $s_{1}<s_{2}<\ldots<s_{m}$; of course this sum vanishes if $m>N$, consistently with Notation 1.1.

The solutions $z_{n} \equiv z_{n}(t)$ of this $N$-body problem are provided-consistently with the expressions (2.1b) — by the $N$ zeros of the following $t$-dependent (monic) polynomial $\psi_{N}(z ; t)$ of degree $N$ in $z$ :

$$
\psi_{N}(z ; t)=z^{N}+\sum_{m=1}^{N}\left[c_{m}(t) z^{N-m}\right]
$$

where the coefficients $c_{m}(t)$ are themselves the solutions of the system of $N$ ODEs

$$
\ddot{c}_{m}=-\omega^{2} c_{m}+2 \sum_{\ell=1, \ell \neq m}^{N}\left(c_{m}-c_{\ell}\right)^{-3} .
$$

Because this is a well-known solvable model, the time-dependence of these $N$ quantities $c_{m}(t)$ can be obtained by solving an algebraic (in fact polynomial) problem, indeed the solution of the initialvalue problem of this dynamical system, (2.3), is provided by the following prescription (see for instance section 4.2.2 in [5] or [4,9]): the $N$ quantities $c_{m} \equiv c_{m}(t)$ are the $N$ eigenvalues of the $N \times N$ (t-dependent) matrix

$$
\mathbf{C}(t)=\mathbf{C}(0) \cos (\omega t)+\dot{\mathbf{C}}(0) \frac{\sin (\omega t)}{\omega},
$$

with

$$
\begin{gathered}
\mathbf{C}(0)=\operatorname{diag}\left[c_{m}(0)\right], \\
\dot{\mathbf{C}}(0)=\operatorname{diag}\left[\dot{c}_{m}(0)\right]+\mathbf{i}[\mathbf{M}(0), \mathbf{C}(0)],
\end{gathered}
$$

where of course (see (2.1b))

$$
\begin{gathered}
c_{m}(0)=(-1)^{m} \sum_{1 \leq s_{1}<s_{2} \ldots<s_{m} \leq N}\left\{\prod_{r=1}^{m}\left[z_{s_{r}}(0)\right]\right\}, \\
\dot{c}_{m}(0)=(-1)^{m} \sum_{1 \leq s_{1}<s_{2} \ldots<s_{m} \leq N} \sum_{q=1}^{m}\left\{\dot{z}_{s_{q}}(0) \prod_{r=1, r \neq q}^{m}\left[z_{s_{r}}(0)\right]\right\},
\end{gathered}
$$

and in the right-hand side of $(2.4 \mathrm{c})$

$$
[\mathbf{M}(0), \mathbf{C}(0)] \equiv \mathbf{M}(0) \mathbf{C}(0)-\mathbf{C}(0) \mathbf{M}(0)
$$

with the matrix $\mathbf{M}(0)$ defined componentwise in terms of the initial data $z_{n}(0)$ as follows:

$$
\begin{aligned}
& M_{n m}(0)=-\left[z_{n}(0)-z_{m}(0)\right]^{-2}, \quad n \neq m, \\
& M_{n n}(0)=-\sum_{\ell=1, \ell \neq n}^{N} M_{n \ell}(0)=\sum_{\ell=1, \ell \neq n}^{N}\left[z_{n}(0)-z_{\ell}(0)\right]^{-2} .
\end{aligned}
$$

Note that these formulas provide an explicit definition of the $N$ time-dependent coefficients $c_{m}(t)$ in terms of the initial data $z_{n}(0), \dot{z}_{n}(0)$ of the $N$-body problem of goldfish type characterized 
by the Newtonian equations of motion (2.1), via algebraic operations, amounting essentially to the solution of polynomial equations of degree $N$; and that the values $z_{n}(t)$ at time $t$ of the particle coordinates $z_{n}$ are then provided by the $N$ zeros of the polynomial $\psi_{N}(z ; t)$, explicitly known (see (2.2)) in terms of its $N$ coefficients $c_{m}(t)$. It is thereby demonstrated that the $N$-body problem of goldfish type characterized by the Newtonian equations of motion (2.1) is solvable (see Remark 1.1).

Remark 2.1. Let us call attention to a (well known) tricky point associated with the solution-as described above - of the $N$-body problem of goldfish type characterized by the Newtonian equations of motion (2.1). The identification of the $N$ eigenvalues of a given matrix is only unique up to permutations, and likewise the identification of the zeros of a polynomial is only unique up to permutations. Therefore the $N$ coordinates $z_{n}=z_{n}(t)$ yielded by the solution detailed above are only identified up to permutations of their $N$ labels $n$. The (only) way to identify a specific coordinatesay, the coordinate $z_{1}(t)$ that corresponds to the initial data $z_{1}(0), \dot{z}_{1}(0)$-is by following the (continuous) time evolution of the coordinate $z_{1}(t)$ from its (assigned) initial value $z_{1}(0)$ to its value $z_{1}(t)$ at time $t$. In this manner one arrives at the uniquely defined value of the coordinate $z_{1}(t)$ corresponding to the initial value $z_{1}(0)$, which coincides of course with that uniquely yielded by the time evolution of the $N$-body problem (2.1). An analogous phenomenology is also relevant to the solution of model (1.1a).

Because of the way system (2.1) is constructed, its equilibria can be obtained by finding the zeros of the polynomials whose coefficients are equilibria of system (2.3). On the other hand, it is known that the zeros of the $N$-th degree Hermite polynomial are equilibria of system (2.3) (see for instance [4]). This relationship allows to study the properties of the polynomials whose coefficients are the zeros of Hermite polynomials.

The findings reported above imply the possibility to display in completely explicit form the solution of the $N$-body problem of goldfish type characterized by the Newtonian equations of motion (2.1) for $N=2,3,4$; but we doubt this would be very illuminating, and we therefore leave this task to the eager reader. We rather like to emphasize that these findings imply that, for arbitrary $N$-and arbitrary positive $\omega$-this $N$-body problem is isochronous, all its solutions satisfying the periodicity property

$$
z_{n}(t+T)=z_{n}(t)
$$

with $T=2 \pi / \omega$ (or, possibly, the period $T$ might be replaced by an integer multiple of $2 \pi / \omega$, generally small with respect to its possible maximal value $N$ !, which is of course a quite large number if $N$ is large: see [6]). We end this paper by displaying a few examples of this phenomenology.

Example 2.1. For $N=2$ and $\omega=1$, taking into account that $c_{1}=-z_{1}-z_{2}$ and $c_{2}=z_{1} z_{2}$, we see that system (2.1) reduces to

$$
\begin{aligned}
& \ddot{z}_{1}=\frac{2 \dot{z}_{1} \dot{z}_{2}}{z_{1}-z_{2}}-\frac{1}{\left(z_{1}-z_{2}\right)}\left[z_{1}^{2}-\frac{2\left(z_{1}-1\right)}{\left(z_{1}+z_{2}+z_{1} z_{2}\right)^{3}}\right], \\
& \ddot{z}_{2}=-\frac{2 \dot{z}_{1} \dot{z}_{2}}{z_{1}-z_{2}}+\frac{1}{\left(z_{1}-z_{2}\right)}\left[z_{2}^{2}-\frac{2\left(z_{2}-1\right)}{\left(z_{1}+z_{2}+z_{1} z_{2}\right)^{3}}\right] .
\end{aligned}
$$




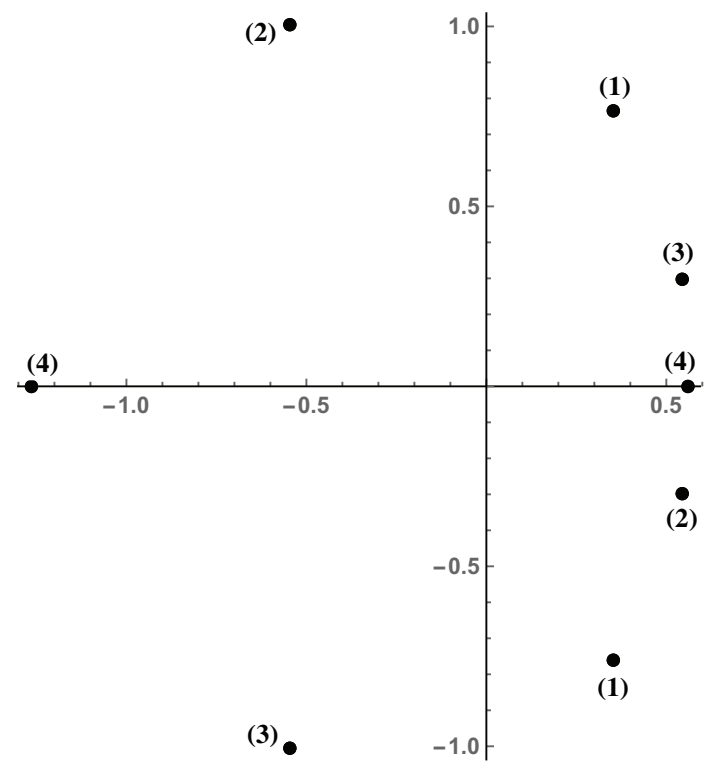

Fig. 1. Equilibria of system (2.6) in the complex $z$-plane. Each equilibrium is represented by the two points $\hat{z}_{1}^{(j)}$ and $\hat{z}_{2}^{(j)}$ labeled by the index $j=1,2,3,4$, see the display (2.6 Equilibria).

The assignment $\omega=1$ is motivated by the fact that this quantity, $\omega$, can be eliminated from system (1.1a) by a constant rescaling of the dependent variables $z_{n}(t)$ and of the independent variable $t$.

System (2.6) has 4 equilibrium configurations $\left(\hat{z}_{1}^{(j)}, \hat{z}_{2}^{(j)}\right), j=1,2,3,4$, up to the exchange of $\hat{z}_{1}^{(j)}$ with $\hat{z}_{2}^{(j)}$, whose approximate numerical values are given below:

$$
\begin{aligned}
& \left(\hat{z}_{1}^{(1)}, \hat{z}_{2}^{(1)}\right)=(0.353553-0.762959 \mathbf{i}, 0.353553+0.762959 \mathbf{i}), \\
& \left(\hat{z}_{1}^{(2)}, \hat{z}_{2}^{(2)}\right)=(-0.54455+1.00281 \mathbf{i}, 0.54455-0.295704 \mathbf{i}), \\
& \left(\hat{z}_{1}^{(3)}, \hat{z}_{2}^{(3)}\right)=(-0.54455-1.00281 \mathbf{i}, 0.54455+0.295704 \mathbf{i}), \\
& \left(\hat{z}_{1}^{(4)}, \hat{z}_{2}^{(4)}\right)=(-1.26575,0.558645) .
\end{aligned}
$$

These equilibria can be obtained either by the substitution $z_{n}(t)=\hat{z}_{n}, n=1,2$ into system (2.6) and the subsequent solution of the resulting system of algebraic equations for $\hat{z}_{1}, \hat{z}_{2}$, or by finding the zeros of the polynomials whose coefficients are the equilibria of system (2.3) for $N=2$. We note that, in this case where $N=2$ and $\omega=1$, system (2.3) has the four equilibria $\left(\frac{1}{\sqrt{2}},-\frac{1}{\sqrt{2}}\right)$, $\left(-\frac{1}{\sqrt{2}}, \frac{1}{\sqrt{2}}\right),\left(\frac{\mathbf{i}}{\sqrt{2}},-\frac{\mathbf{i}}{\sqrt{2}}\right)$, and $\left(-\frac{\mathbf{i}}{\sqrt{2}}, \frac{\mathbf{i}}{\sqrt{2}}\right)$. Two of them are the zeros $\pm \frac{1}{\sqrt{2}}$ of the Hermite polynomial $H_{2}(c)=4 c^{2}-2$, which is consistent with the known fact that the zeros of the $N$-th order Hermite polynomial are equilibria of system (2.3).

In Fig. 1 each equilibrium of system (2.6) is represented by the two points $\hat{z}_{1}^{(j)}$ and $\hat{z}_{2}^{(j)}$, labeled by $(j)$, where $j=1,2,3,4$.

Below we provide graphs of the real and imaginary parts of the components of the solution $\left(z_{1}(t), z_{2}(t)\right)$ of system (2.6) as functions of time, and some trajectories of the particles $z_{1}$ and $z_{2}$ in the complex $z$-plane. These graphs have been obtained by numerical integration of system (2.6), using Mathematica 10. We employed the command NDSolve with the automatic choice of the method and the accuracy. The program script is provided in the Appendix. 
O. Bihun, F. Calogero / A new solvable many-body problem of goldfish type

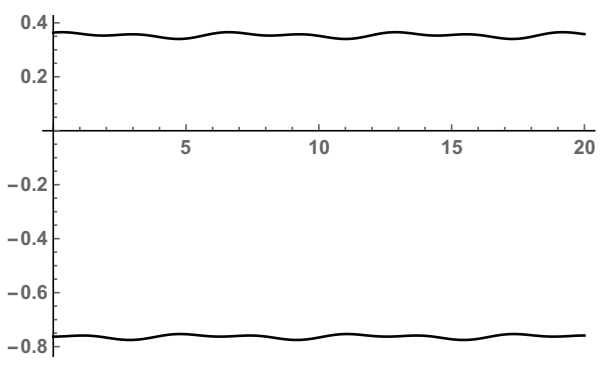

Fig. 2. System (2.6), initial conditions (2.6a). Graphs of the real (upper curve) and imaginary (lower curve) parts of the coordinate $z_{1}(t)$; period $2 \pi$.

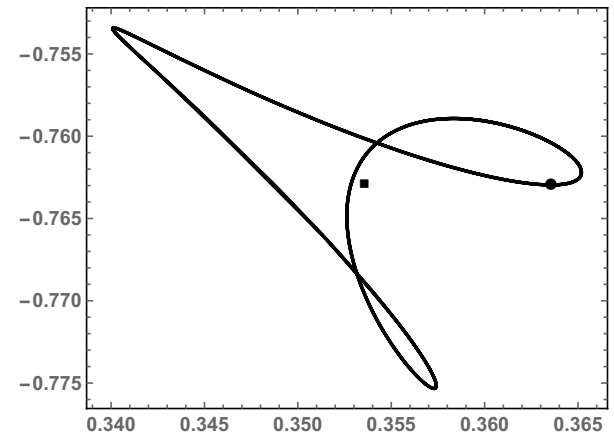

Fig. 3. System (2.6), initial conditions (2.6a). Trajectory, in the complex $z$-plane, of $z_{1}(t)$. The square respectively the dot indicate the positions of $\hat{z}_{1}^{(1)}$ respectively $z_{1}(0)$, i.e. of a nearby equilibrium point respectively the initial value of $z_{1}(t)$; period $2 \pi$.

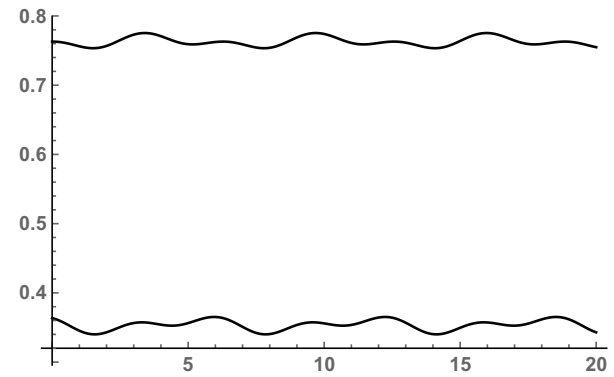

Fig. 4. System (2.6), initial conditions (2.6a). Graphs of the real (lower curve) and the imaginary (upper curve) parts of the coordinate $z_{2}(t)$; period $2 \pi$.

(2.6a) Initial conditions $z_{1}(0)=\hat{z}_{1}^{(1)}+0.01, z_{2}(0)=\hat{z}_{2}^{(1)}+0.01$, $\dot{z}_{1}(0)=0.01, \dot{z}_{2}(0)=-0.01$. See Fig. 2, 3, 4, 5 .

(2.6b) Initial conditions $z_{1}(0)=\hat{z}_{1}^{(1)}+0.01+0.01 \mathbf{i}, z_{2}(0)=\hat{z}_{2}^{(1)}+0.01+0.01 \mathbf{i}$, $\dot{z}_{1}(0)=-0.01, \dot{z}_{2}(0)=0.01$. See Fig. 6,7 .

(2.6c) Initial conditions $z_{1}(0)=\hat{z}_{1}^{(2)}+0.01+0.01 \mathbf{i}, z_{2}(0)=\hat{z}_{2}^{(2)}+0.01+0.01 \mathbf{i}$, $\dot{z}_{1}(0)=-0.01, \dot{z}_{2}(0)=0.01$. See Fig. 8,9 . 
O. Bihun, F. Calogero / A new solvable many-body problem of goldfish type

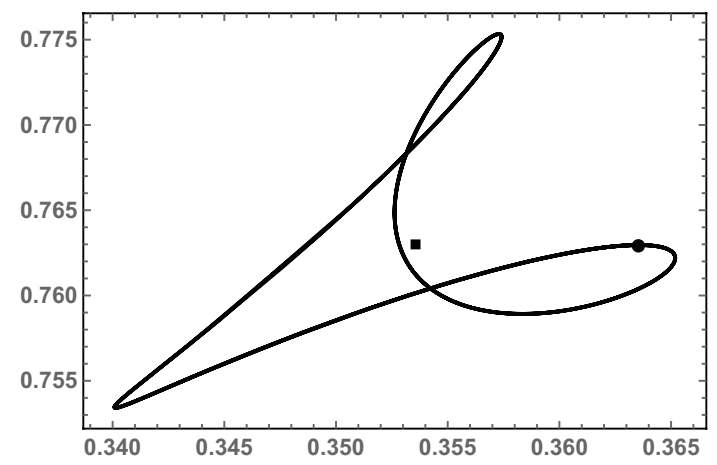

Fig. 5. System (2.6), initial conditions (2.6a). Trajectory, in the complex $z$-plane, of $z_{2}(t)$. The square respectively the dot indicate the positions of $\hat{z}_{2}^{(1)}$ respectively $z_{2}(0)$, i.e of a nearby equilibrium point respectively the initial value of $z_{2}(t)$; period $2 \pi$.

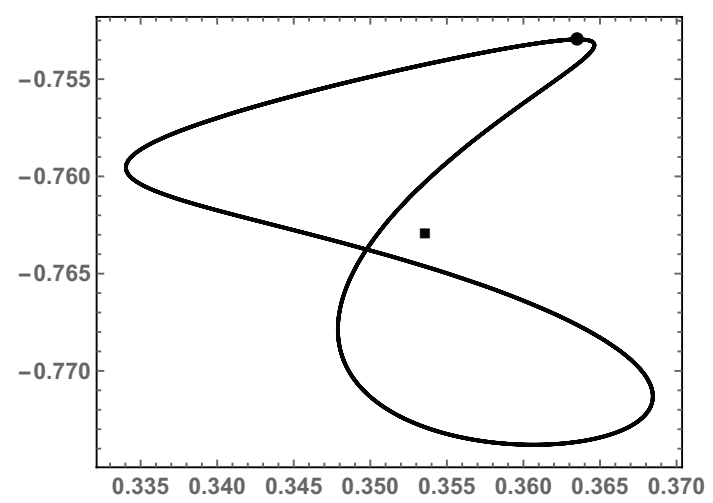

Fig. 6. System (2.6), initial conditions (2.6b). Trajectory, in the complex $z$-plane, of $z_{1}(t)$. The square respectively the dot indicate the positions of $\hat{z}_{1}^{(1)}$ respectively $z_{1}(0)$, i.e. of a nearby equilibrium point respectively the initial value of $z_{1}(t)$; period $2 \pi$.

(2.6d) Initial conditions $z_{1}(0)=\hat{z}_{1}^{(3)}+0.01+0.01 \mathbf{i}, z_{2}(0)=\hat{z}_{2}^{(3)}+0.01+0.01 \mathbf{i}$, $\dot{z}_{1}(0)=-0.005, \dot{z}_{2}(0)=0.005$. See Fig. 10 and 11 .

(2.6e) Initial conditions $z_{1}(0)=\hat{z}_{1}^{(4)}+0.01+0.01 \mathbf{i}, z_{2}(0)=\hat{z}_{2}^{(4)}+0.01+0.01 \mathbf{i}$, $\dot{z}_{1}(0)=-0.01, \dot{z}_{2}(0)=0.01$. See Fig. 10 and 11 . 
O. Bihun, F. Calogero / A new solvable many-body problem of goldfish type

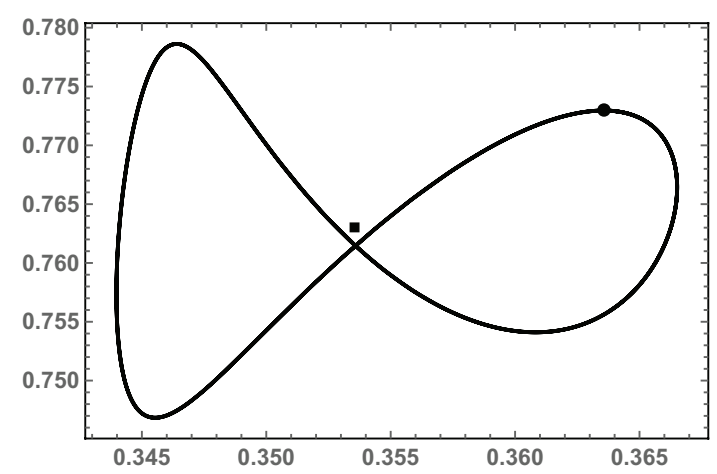

Fig. 7. System (2.6), initial conditions (2.6b). Trajectory, in the complex $z$-plane, of $z_{2}(t)$. The square respectively the dot indicate the positions of $\hat{z}_{2}^{(1)}$ respectively $z_{2}(0)$, i.e. of a nearby equilibrium point respectively the initial value of $z_{2}(t)$; period $2 \pi$.

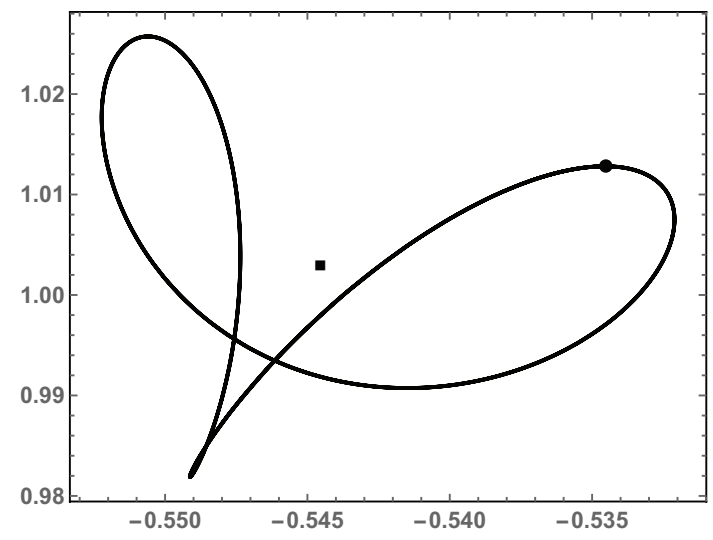

Fig. 8. System (2.6), initial conditions (2.6c). Trajectory, in the complex $z$-plane, of $z_{1}(t)$. The square respectively the dot indicate the positions of $\hat{z}_{1}^{(2)}$ respectively $z_{1}(0)$, i.e. of a nearby equilibrium point respectively the initial value of $z_{1}(t)$; period $2 \pi$.

Example 2.2. For $N=3$ and $\omega=1$, taking into account that $c_{1}=-z_{1}-z_{2}-z_{3}, c_{2}=z_{1} z_{2}+z_{1} z_{3}+$ $z_{2} z_{3}$, and $c_{3}=-z_{1} z_{2} z_{3}$, we see that system (2.1) reduces to

$$
\begin{aligned}
& \ddot{z}_{1}=\frac{2 \dot{z}_{1} \dot{z}_{2}}{z_{1}-z_{2}}+\frac{2 \dot{z}_{1} \dot{z}_{3}}{z_{1}-z_{3}} \\
& \left.-\frac{1}{\left(z_{1}-z_{2}\right)\left(z_{1}-z_{3}\right)}\left[z_{1}^{2} F_{1}\left(z_{1}, z_{2}, z_{3}\right)+z_{1} F_{2}\left(z_{1}, z_{2}, z_{3}\right)+F_{3}\left(z_{1}, z_{2}, z_{3}\right)\right)\right], \\
& \ddot{z}_{2}=-\frac{2 \dot{z}_{1} \dot{z}_{2}}{z_{1}-z_{2}}+\frac{2 \dot{z}_{2} \dot{z}_{3}}{z_{2}-z_{3}} \\
& \left.+\frac{1}{\left(z_{1}-z_{2}\right)\left(z_{2}-z_{3}\right)}\left[z_{2}^{2} F_{1}\left(z_{1}, z_{2}, z_{3}\right)+z_{2} F_{2}\left(z_{1}, z_{2}, z_{3}\right)+F_{3}\left(z_{1}, z_{2}, z_{3}\right)\right)\right], \\
& \ddot{z}_{3}=-\frac{2 \dot{z}_{1} \dot{z}_{3}}{z_{1}-z_{3}}-\frac{2 \dot{z}_{2} \dot{z}_{3}}{z_{2}-z_{3}} \\
& \left.-\frac{1}{\left(z_{1}-z_{3}\right)\left(z_{2}-z_{3}\right)}\left[z_{3}^{2} F_{1}\left(z_{1}, z_{2}, z_{3}\right)+z_{3} F_{2}\left(z_{1}, z_{2}, z_{3}\right)+F_{3}\left(z_{1}, z_{2}, z_{3}\right)\right)\right],
\end{aligned}
$$

Co-published by Atlantis Press and Taylor \& Francis 
O. Bihun, F. Calogero / A new solvable many-body problem of goldfish type

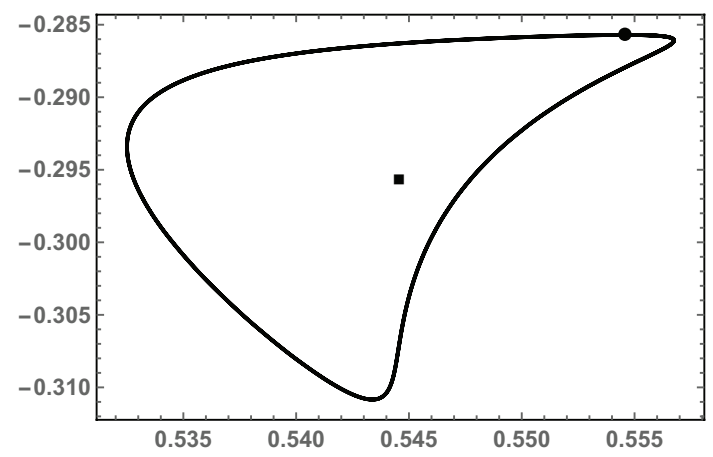

Fig. 9. System (2.6), initial conditions (2.6c). Trajectory, in the complex $z$-plane, of $z_{2}(t)$. The square respectively the dot indicate the positions of $\hat{z}_{2}^{(2)}$ respectively $z_{2}(0)$, i.e. of a nearby equilibrium point respectively the initial value of $z_{2}(t)$; period $2 \pi$.

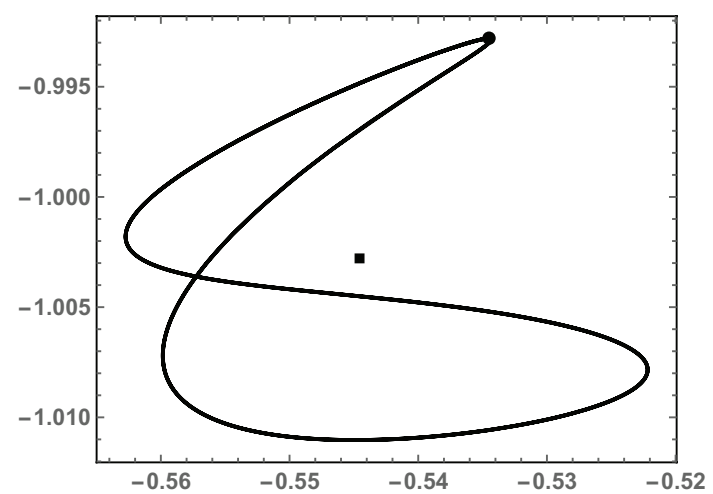

Fig. 10. System (2.6), initial conditions (2.6d). Trajectory, in the complex z-plane, of $z_{1}(t)$. The square respectively the dot indicate the positions of $\hat{z}_{1}^{(3)}$ respectively $z_{1}(0)$, i.e. of a nearby equilibrium point respectively the initial value of $z_{1}(t)$; period $2 \pi$.

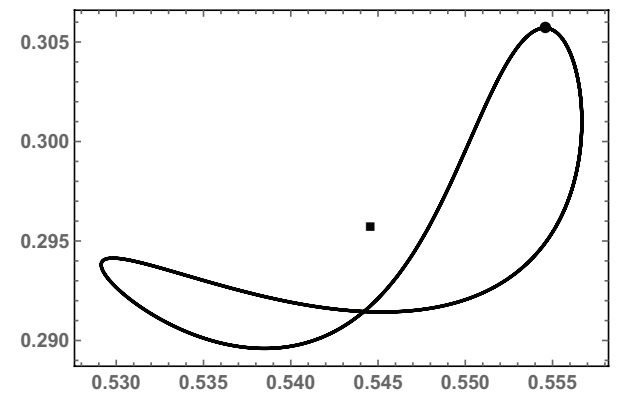

Fig. 11. System (2.6), initial conditions (2.6d). Trajectory, in the complex $z$-plane, of $z_{2}(t)$. The square respectively the dot indicate the positions of $\hat{z}_{2}^{(3)}$ respectively $z_{2}(0)$ of a nearby equilibrium point respecttively the initial value of $z_{2}(t)$; period $2 \pi$. 
O. Bihun, F. Calogero / A new solvable many-body problem of goldfish type

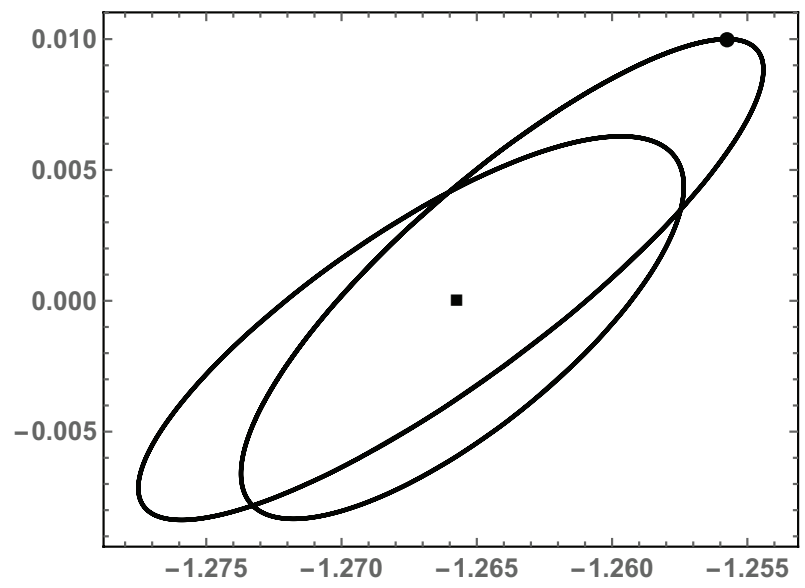

Fig. 12. System (2.6), initial conditions (2.6e). Trajectory, in the complex $z$-plane, of $z_{1}(t)$. The square respectively the dot indicate the positions of $\hat{z}_{1}^{(4)}$ respectively $z_{1}(0)$, i.e. of a nearby equilibrium point respectively the initial value of $z_{1}(t)$; period $2 \pi$.

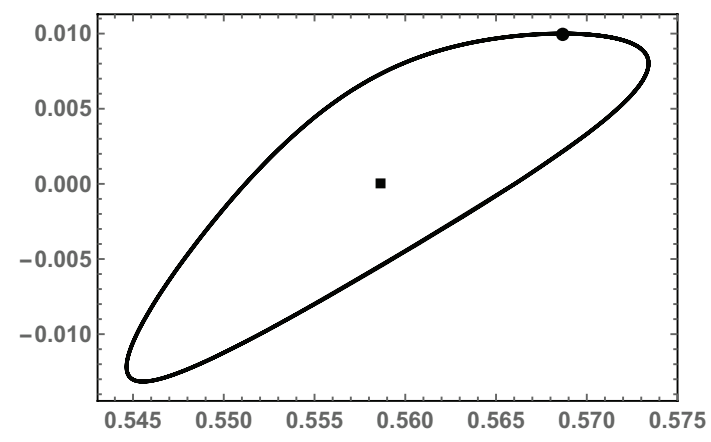

Fig. 13. System (2.6), initial conditions (2.6e). Trajectory, in the complex $z$-plane, of $z_{2}(t)$. The square respectively the dot indicate the positions of $\hat{z}_{2}^{(4)}$ respectively $z_{2}(0)$, i.e. of a nearby equilibrium point respectively the initial value of $z_{2}(t)$; period $2 \pi$.

where

$$
\begin{aligned}
& F_{1}\left(z_{1}, z_{2}, z_{3}\right)=z_{1}+z_{2}+z_{3}-\frac{2}{\left(z_{1}+z_{2}+z_{3}-z_{1} z_{2} z_{3}\right)^{3}} \\
& -\frac{2}{\left(z_{1}+z_{2}+z_{3}+z_{1} z_{2}+z_{1} z_{3}+z_{2} z_{3}\right)^{3}}, \\
& F_{2}\left(z_{1}, z_{2}, z_{3}\right)=-z_{1} z_{2}-z_{1} z_{3}-z_{2} z_{3} \\
& +\frac{2}{\left(z_{1}+z_{2}+z_{3}+z_{1} z_{2}+z_{1} z_{3}+z_{2} z_{3}\right)^{3}}+\frac{2}{\left(z_{1} z_{2}+z_{1} z_{3}+z_{2} z_{3}+z_{1} z_{2} z_{3}\right)^{3}}, \\
& F_{3}\left(z_{1}, z_{2}, z_{3}\right)=z_{1} z_{2} z_{3} \\
& +\frac{2}{\left(z_{1}+z_{2}+z_{3}-z_{1} z_{2} z_{3}\right)^{3}}-\frac{2}{\left(z_{1} z_{2}+z_{1} z_{3}+z_{2} z_{3}+z_{1} z_{2} z_{3}\right)^{3}} .
\end{aligned}
$$

Co-published by Atlantis Press and Taylor \& Francis 
We obtained equilibria of system (2.7a), (2.7b) as follows. First, we found the equilibria of system (2.3) for $N=3$; they are given by $\left(0, \sqrt{\frac{3}{2}},-\sqrt{\frac{3}{2}}\right)$ and $\left(0, \mathbf{i} \sqrt{\frac{3}{2}},-\mathbf{i} \sqrt{\frac{3}{2}}\right)$, up to the permutations of the three coordinates. Second, we found the zeros of the monic polynomials whose coefficients are the equilibria of system (2.3) for $N=3$. These zeros are equilibriumum solutions of system (2.1) because of how this system is constructed. Therefore, system (2.7a), (2.7b) has at least 12 equilibrium configurations $\left(\hat{z}_{1}^{(j)}, \hat{z}_{2}^{(j)}, \hat{z}_{3}^{(j)}\right), j=1,2, \ldots, 12$, up to the permutations of $\hat{z}_{1}^{(j)}, \hat{z}_{2}^{(j)}$ and $\hat{z}_{3}^{(j)}$, whose approximate numerical values are given below:

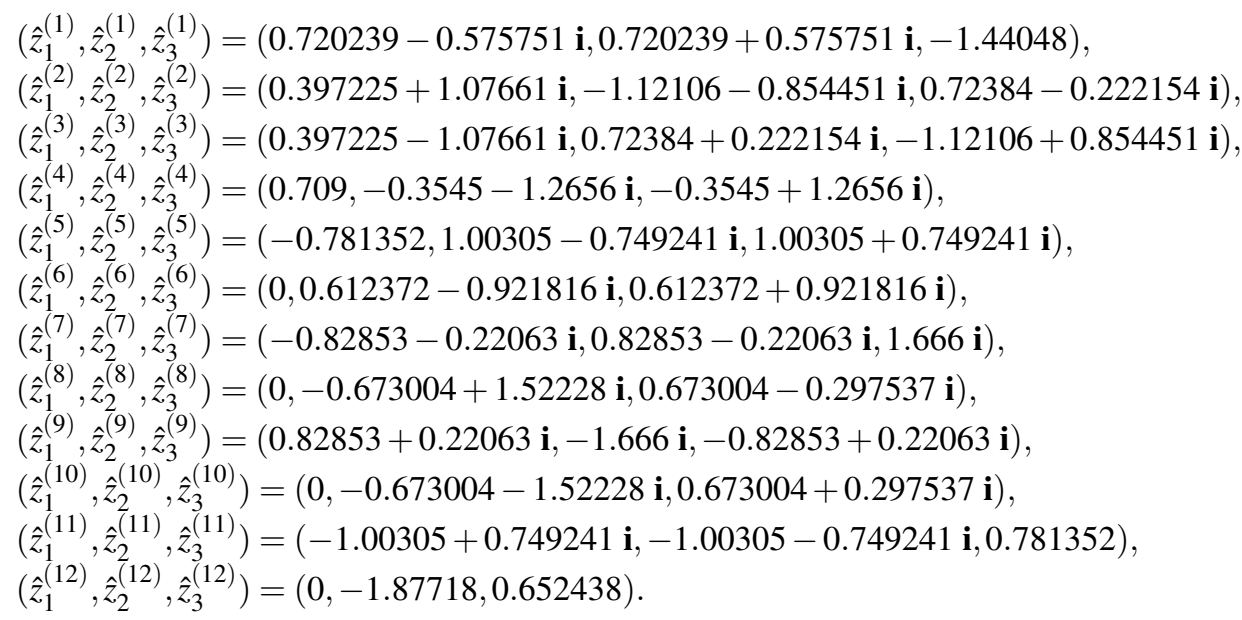

It is possible that the system of algebraic equations characterizing the equilibria of (2.7a), (2.7b) has additional solutions besides those listed above. A direct attempt to solve this system of algebraic equations using Mathematica 10 was unsuccessful, and we did not deem the matter sufficiently relevant to justify further investigations.

In Fig. 14 each equilibrium of system $(2.7 \mathrm{a}),(2.7 \mathrm{~b})$ is represented by the three points $\hat{z}_{1}^{(j)}, \hat{z}_{2}^{(j)}$ and $\hat{z}_{3}^{(j)}$, labeled by the index $j$, where $j=1,2, \ldots, 12$.

Below we provide some trajectories, in the complex plane, of the particles $z_{1}, z_{2}$ and $z_{3}$ whose evolution is described by system $(2.7 \mathrm{a}),(2.7 \mathrm{~b})$ with the initial conditions

$$
\begin{gathered}
z_{1}(0)=\hat{z}_{1}^{(3)}+0.3+0.3 \mathbf{i}, \dot{z}_{1}(0)=0.1 \\
z_{2}(0)=\hat{z}_{2}^{(3)}+0.1, \dot{z}_{2}(0)=0.1+0.1 \mathbf{i}, \\
z_{3}(0)=\hat{z}_{3}^{(3)}+0.1, \dot{z}_{3}(0)=0.1,
\end{gathered}
$$

see Fig. 15, 16 and 17. These graphs have been obtained by numerical integration of system (2.7a), (2.7b) with the initial conditions (2.7c), using Mathematica 10. We employed the command NDSolve with the automatic choice of the method and the accuracy. The program script is provided in the Appendix. 


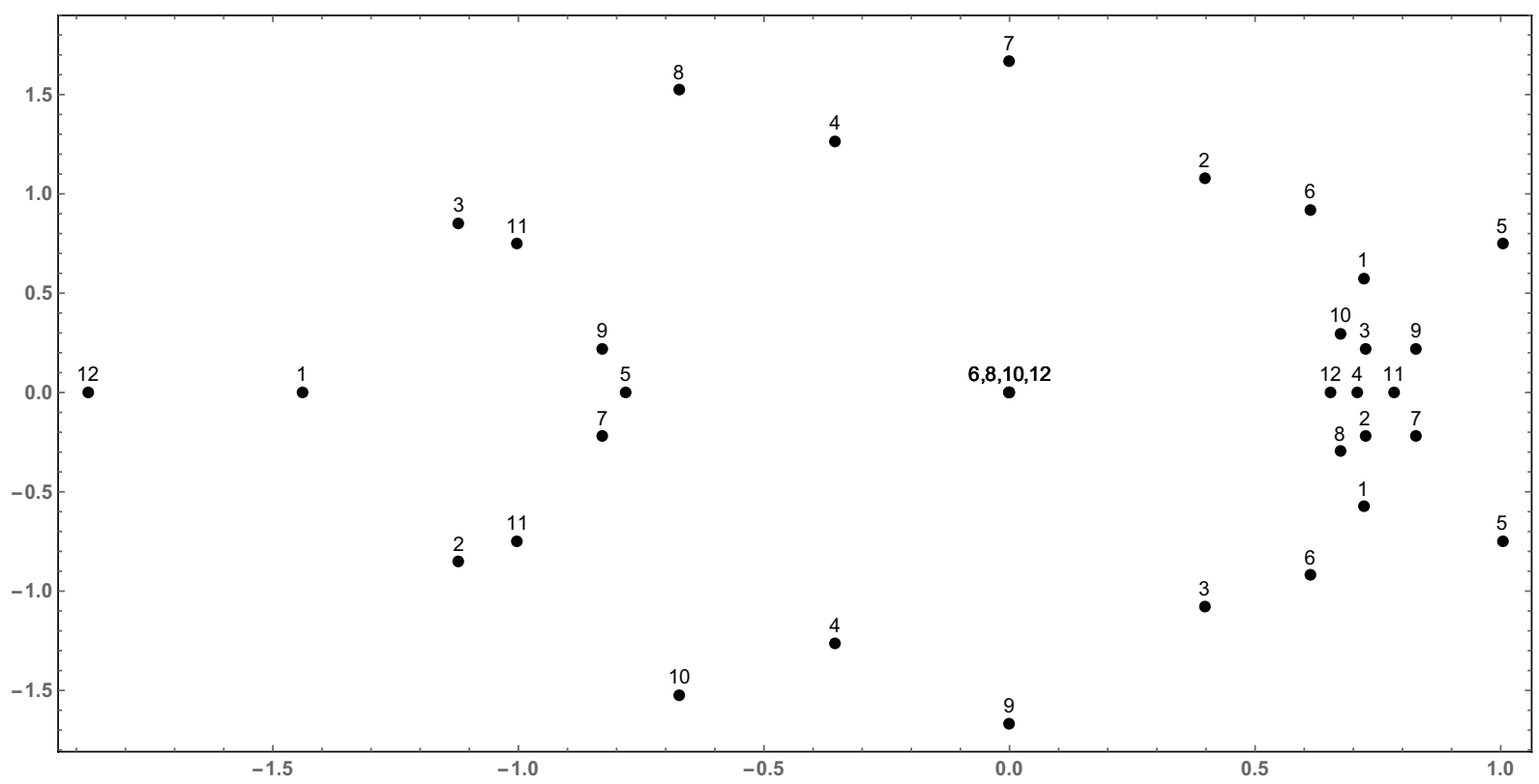

Fig. 14. Equilibria of system (2.7a), (2.7b) in the complex $z$-plane. Each equilibrium is represented by the three points $\hat{z}_{1}^{(j)}, \hat{z}_{2}^{(j)}$ and $\hat{z}_{3}^{(j)}$ indexed and labeled by $j=1,2, \ldots, 12$, see display ( 2.7 Equilibria).

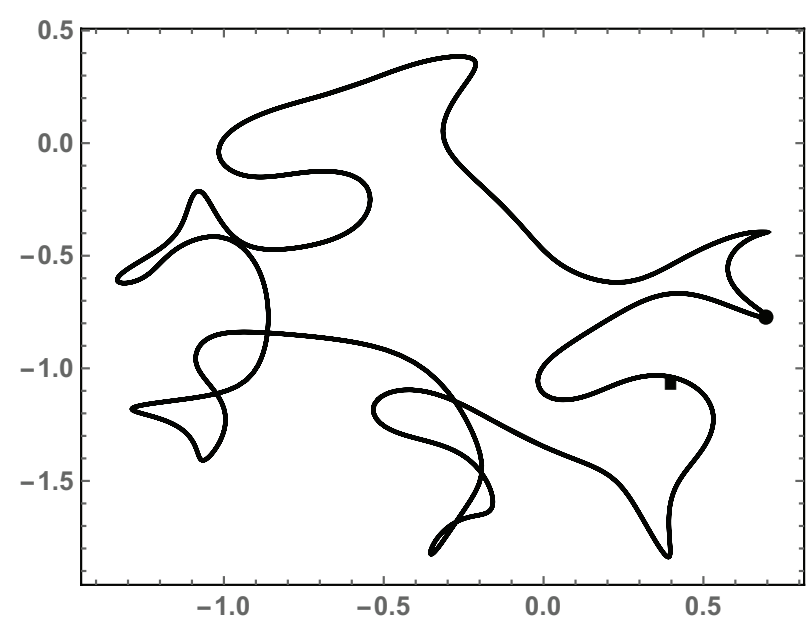

Fig. 15. System (2.7a), (2.7b), initial conditions (2.7c). Trajectory, in the complex $z$-plane, of $z_{1}(t)$. The square respectively the dot indicate the positions of $\hat{z}_{1}^{(3)}$ respectively $z_{1}(0)$, i.e. of a nearby equilibrium point respectively the initial value of $z_{1}(t)$; period $6 \pi$.

\section{Outlook}

The interest of the many-body model introduced and discussed above is demonstrated by its solvable and isochronous character as well as by the remarkable trajectories it features - already in the simple $N=2$ and $N=3$ cases, as shown by the graphs reported above. It is also amusing to observeas the cognoscienti will have noted - that this solvable model has been obtained by appropriately 


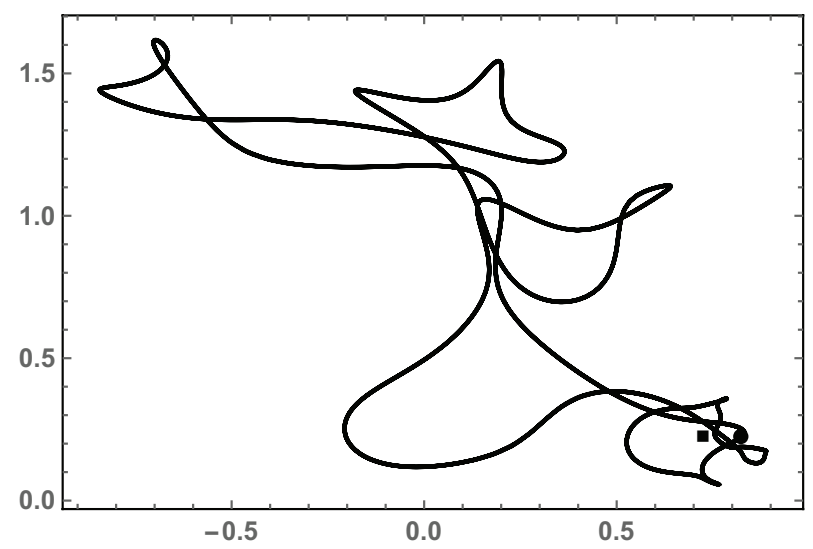

Fig. 16. System (2.7a), (2.7b), initial conditions (2.7c). Trajectory, in the complex $z$-plane, of $z_{2}(t)$. The square respectively the dot indicate the positions of $\hat{z}_{2}^{(3)}$ respectively $z_{2}(0)$, i.e. of a nearby equilibrium point respectively the initial value of $z_{2}(t)$; period $6 \pi$.

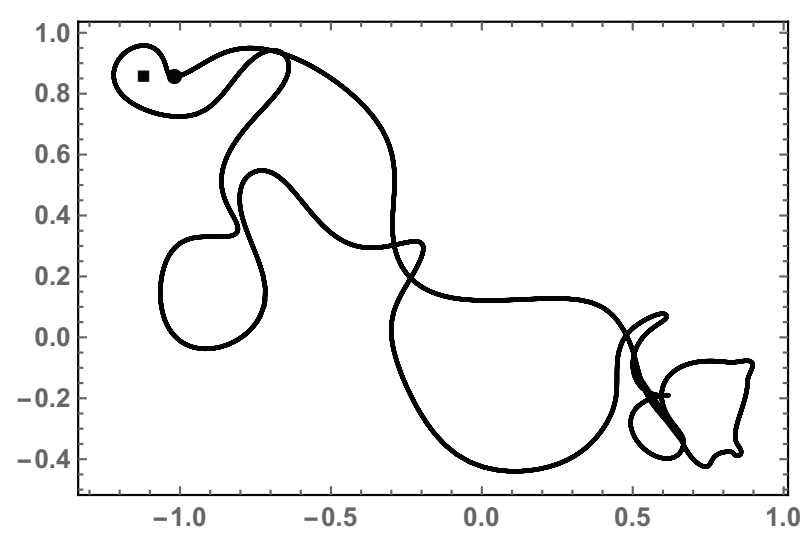

Fig. 17. System (2.7a), (2.7b), initial conditions (2.7c). Trajectory, in the complex $z$-plane, of $z_{3}(t)$. The square respectively the dot indicate the positions of $\hat{z}_{3}^{(3)}$ respectively $z_{3}(0)$, i.e. of a nearby equilibrium point respectively the initial value of $z_{3}(t)$; period $6 \pi$.

combining the two solvable equations-see (1.1a) and (2.3) - which are the prototypes of two, quite different, basic families of solvable many-body problems of Newtonian type. Moreover, the findings reported above provide the point of departure to obtain Diophantine properties of the $N$ zeros of each of the $N$ ! (monic) polynomials the coefficients of which are the $N$ zeros of the Hermite polynomial $H_{N}(z)$ of degree $N$ (these $N$ ! polynomials correspond of course to the $N$ ! permutations of the $N$ zeros of $H_{N}(z)$ ). And a rather ample vista of further developments is provided by these findings.

\section{Acknowledgements}

One of the authors (OB) would like to thank for the hospitality of the University of Rome "La Sapienza" during her visit in the summer 2015. 


\section{Appendix. Scripts to Plot Approximate Solutions of Systems (2.6) and (2.7a), (2.7b)}

\subsection{A Mathematica Script to Plot Approximate Solutions of System (2.6)}

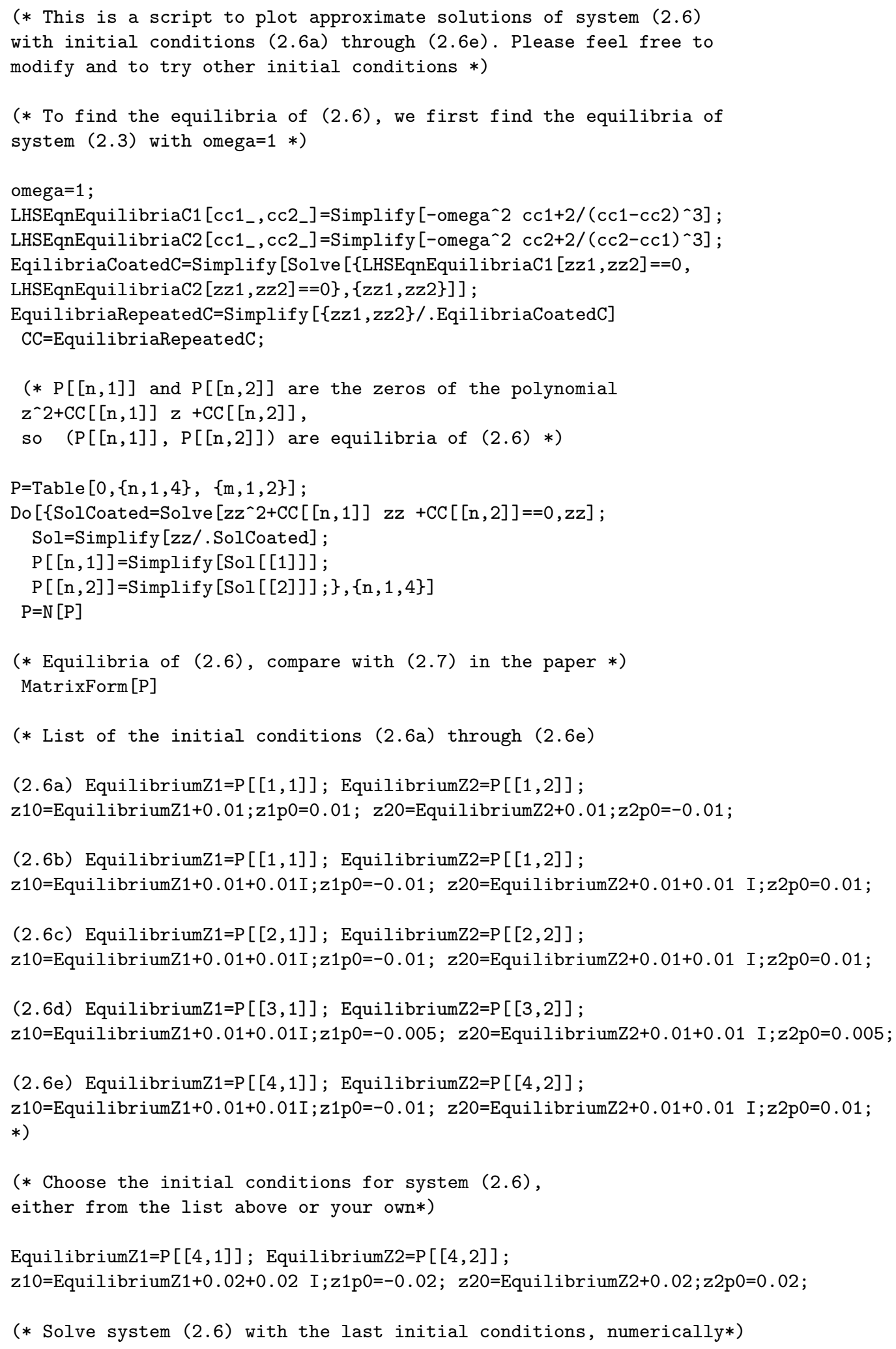




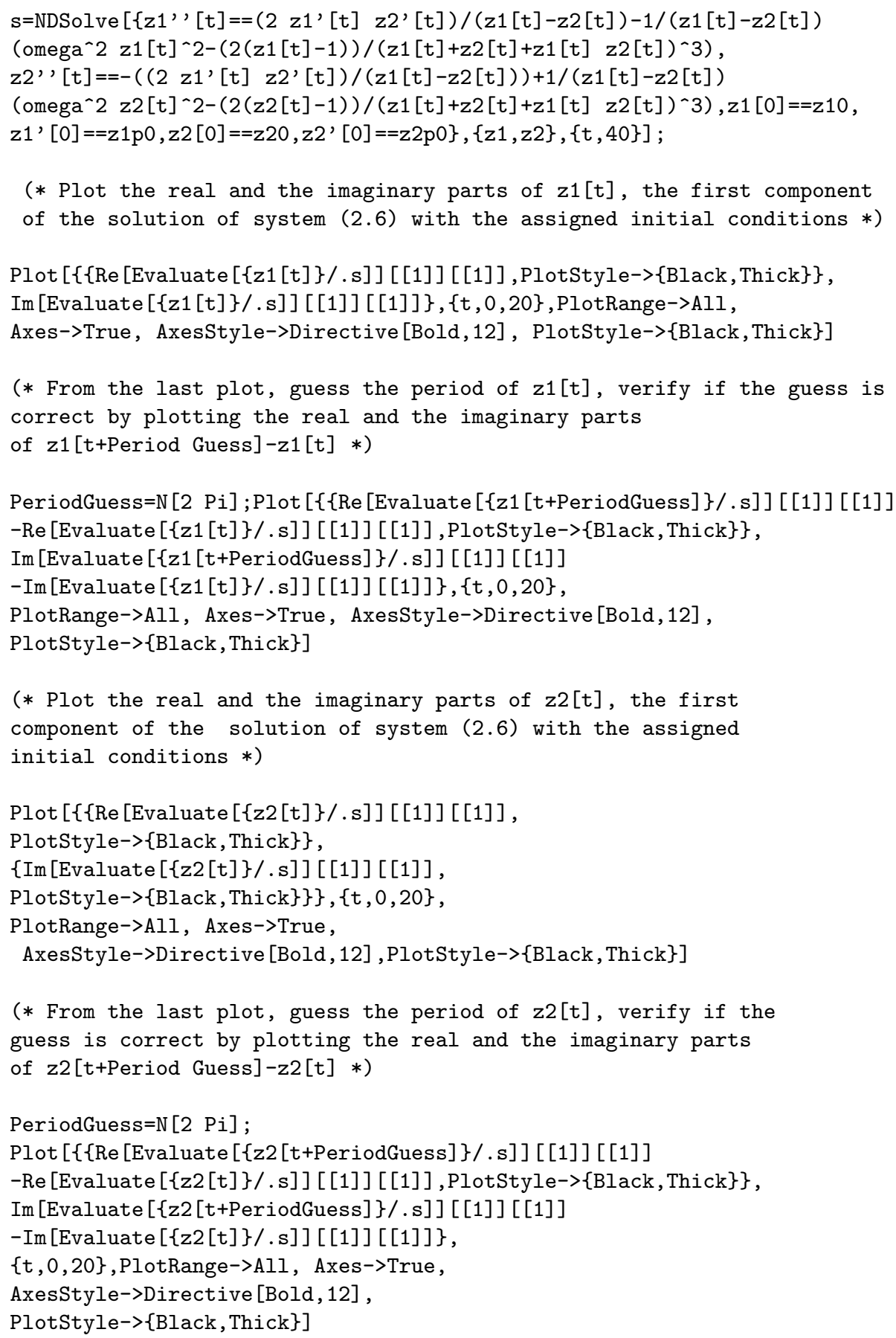


Show [Graph2, Graphics [

\{PointSize [Large],

Point [\{Re [EquilibriumZ1] , Im[EquilibriumZ1]\}],

Point $[\{\operatorname{Re}[z 10], \operatorname{Im}[z 10]\}]$

\}] ]

(* Plot the trajectory, in the complex $z$-plane, of the solution $z 2[t]$ of system (2.6) with the assigned initial conditions *)

Graph3=ParametricPlot [\{Re[Evaluate $[\{z 2[t]\} / . s]][[1]][[1]]$,

$\operatorname{Im}[$ Evaluate $[\{z 2[t]\} / . s]][[1]][[1]]\},\{t, 0,20\}, P l o t R a n g e->A l l$,

Axes->True,

AxesStyle->Directive[Bold,12],PlotStyle->\{Black, Thick\},

AspectRatio->1/1.5];

Show [Graph3, Graphics [

\{PointSize [Large] , Point [\{Re [EquilibriumZ2] , Im[EquilibriumZ2]\}],

Point $[\{\operatorname{Re}[z 20], \operatorname{Im}[z 20]\}]$

\}] ]

\subsection{A Mathematica Script to Plot Approximate Solutions of System (2.7a), (2.7b)}

(* This is a script to plot approximate solutions of system (2.7ab) with initial conditions (2.7c). Please feel free to modify and to try other initial conditions $*$ )

$\mathrm{c} 1=(-\mathrm{z} 1-\mathrm{z} 2-\mathrm{z} 3)$;

$c 2=z 1 \quad z 2+z 1 \quad z 3+z 2 \quad z 3 ;$

$c 3=-z 1 \quad z 2 \quad z 3$

(* To find (some of the) equilibria of system (2.7ab),

we first find the equilibria of system (2.3) for

$\mathrm{N}=3$ and omega $=1$ and then find the zeros of the

monic polynomials whose coefficients are equilbria of $(2.3) *$ )

FF1 $\left[z 1_{-}, z 2_{-}, z 3_{-}\right]=$Simplify $\left[-c 1+2 /(c 1-c 2)^{\wedge} 3+2 /(c 1-c 3)^{\wedge} 3\right]$

FF2 $\left[z 1_{-}, z 2_{-}, z 3_{-}\right]=$Simplify $[-c 2+2 /(c 2-c 1) \wedge 3+2 /(c 2-c 3) \wedge 3]$;

FF3 $\left[z 1_{-}, z 2_{-}, z 3_{-}\right]=$Simplify $[-c 3+2 /(c 3-c 1) \wedge 3+2 /(c 3-c 2) \wedge 3]$

omega=1;

LHSEqnEquilibriaC1 $\left[c c 1_{-}, c c 2_{-}, c c 33_{-}\right]=\operatorname{Simplify}\left[-c c 1+2 /(\mathrm{cc1}-\mathrm{cc} 2)^{\wedge} 3+2 /(\mathrm{cc1} 1-\mathrm{cc} 3)^{\wedge} 3\right]$;

LHSEqnEquilibriaC2 $\left[c c 1_{-}, c c 2_{-}, c c 3_{-}\right]=$Simplify $[-c c 2+2 /(c c 2-c c 1) \wedge 3+2 /(c c 2-c c 3) \wedge 3]$;

LHSEqnEquilibriaC3 [cc1 , cc2 , cc3_] =Simplify $[-c c 3+2 /(c c 3-c c 1) \wedge 3+2 /(c c 3-c c 2) \wedge 3]$;

EqilibriaCoatedC=Simplify [Solve [\{LHSEnEquilibriaC1 $[z z 1, z z 2, z z 3]==0$,

LHSEqnEquilibriaC2 $[z z 1, z z 2, z z 3]==0$,

LHSEqnEquilibriaC3 $[z z 1, z z 2, z z 3]==0\},\{z z 1, z z 2, z z 3\}]]$;

EquilibriaRepeatedC=Simplify $[\{z z 1, z z 2, z z 3\} /$. EqilibriaCoatedC $]$

$\mathrm{CC}=$ EquilibriaRepeatedC;

(* $P[[n, 1]], P[[n, 2]]$ and $P[[n, 3]]$ are the zeros of the polynomial $z^{\wedge} 3+\operatorname{CC}[[n, 1]] \quad z^{\wedge} 2+\operatorname{CC}[[n, 2]] \quad z+\operatorname{CC}[[n, 2]]$,

so (P[[n,1]], $P[[n, 2]], P[[n, 3]]$ )

are equilibria of $(2.7 \mathrm{ab}) *$ ) 


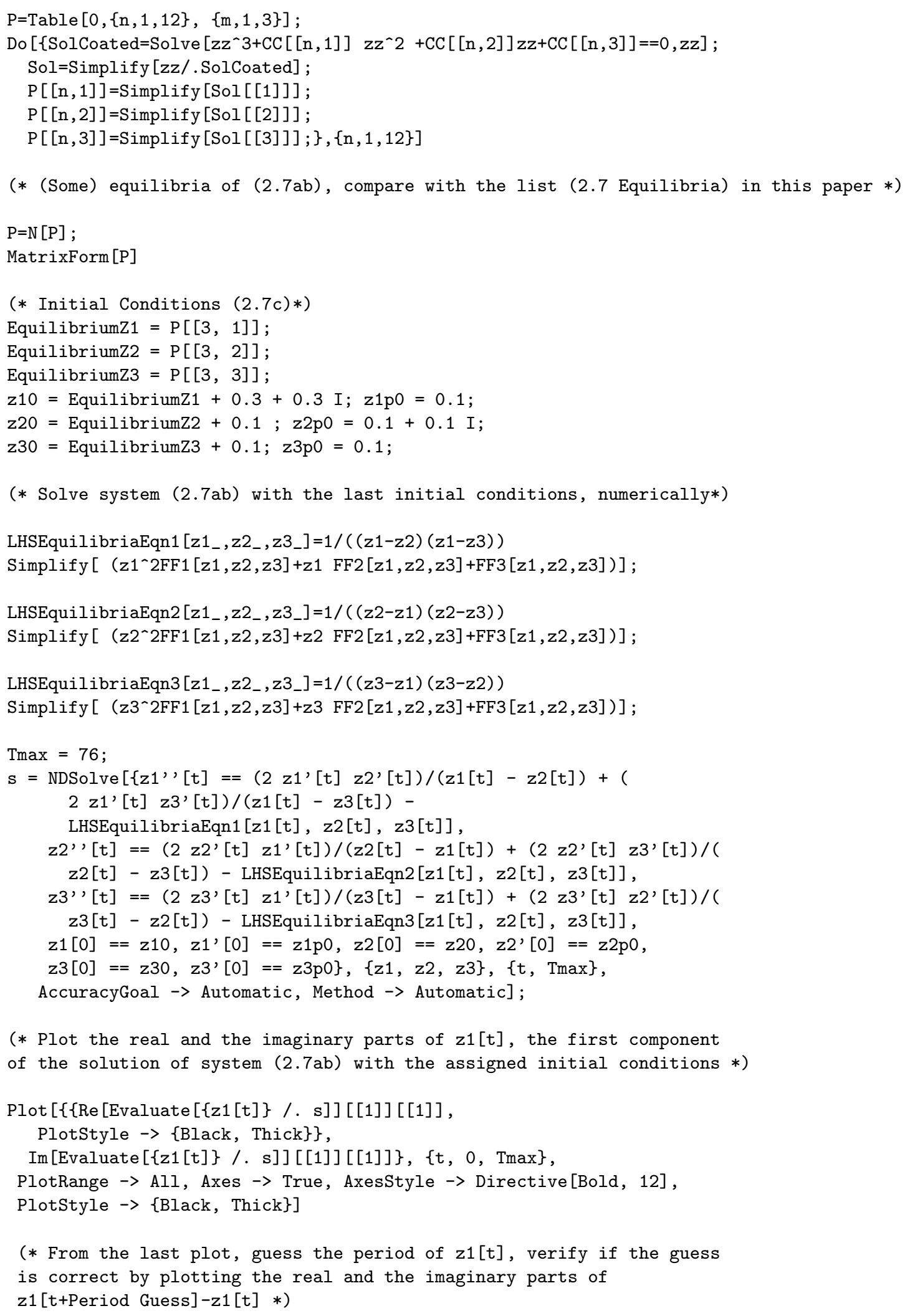




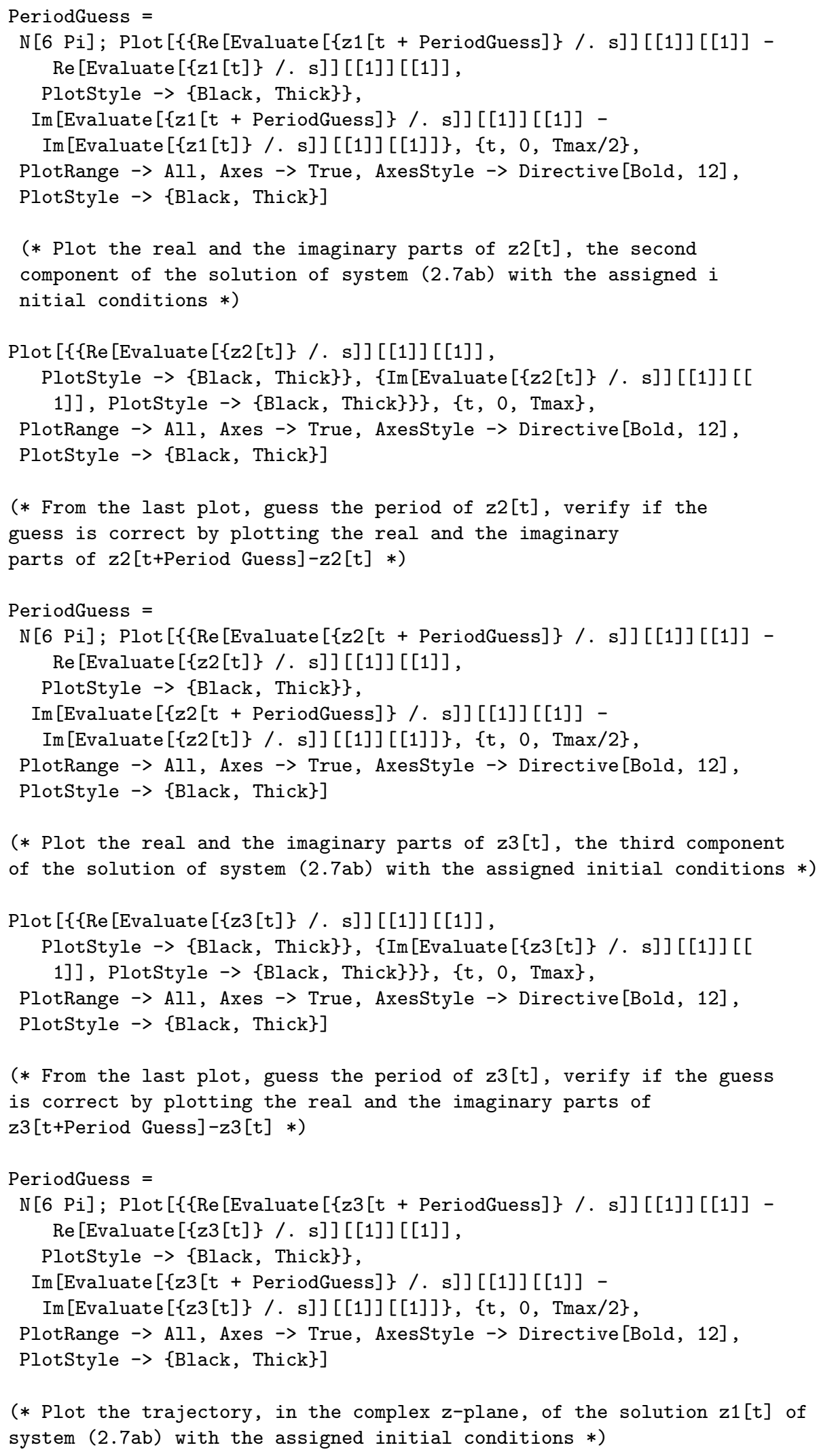




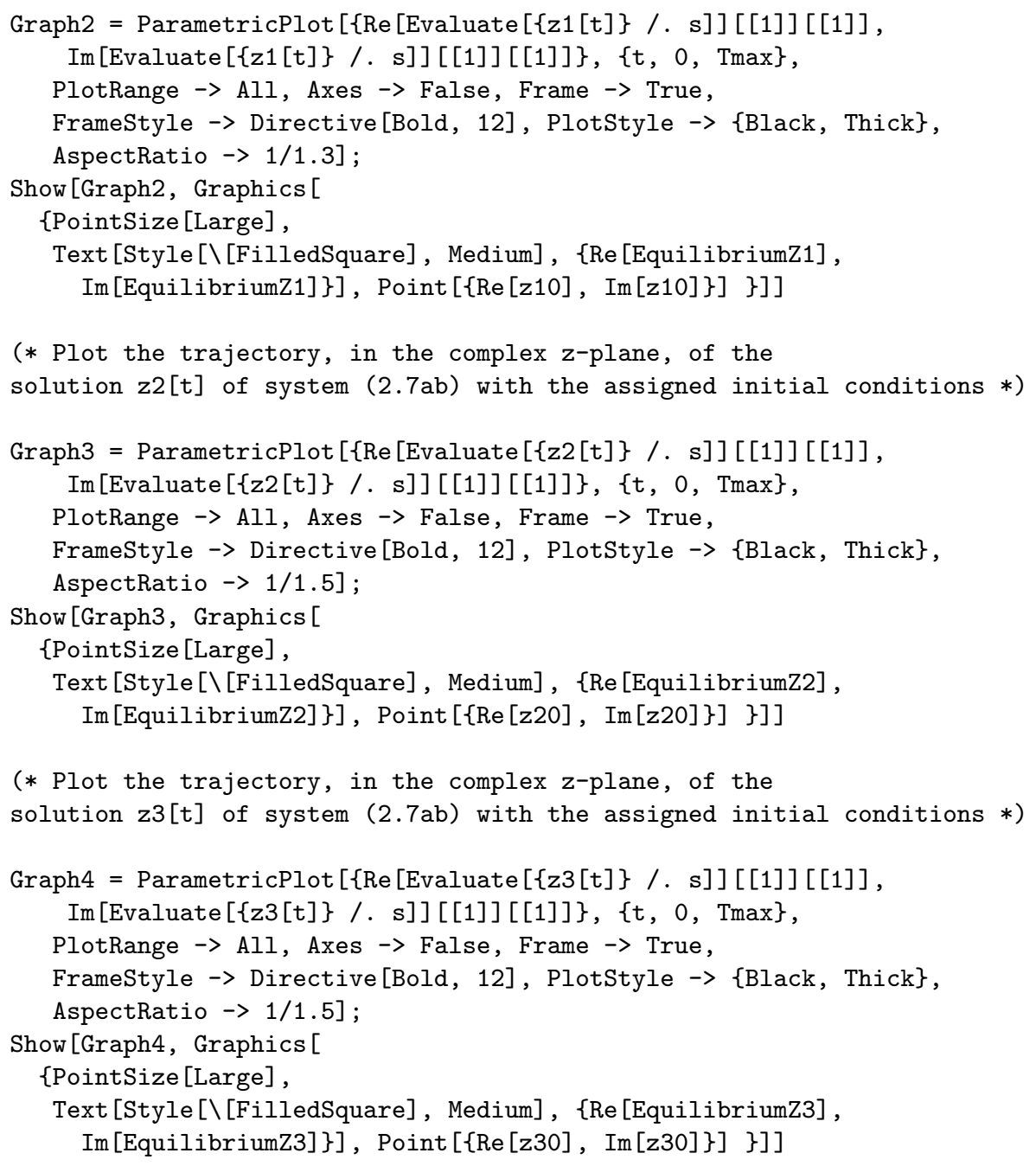

\section{References}

[1] O. Bihun and F. Calogero, Solvable Many-body Models of Goldfish Type with One-, Two- and Threebody Forces, SIGMA 9 (2013), 059-077.

[2] F. Calogero, The "neatest" many-body problem amenable to exact treatments (a "goldfish"?), Physica $\boldsymbol{D}$ 152-153 (2001) 78-84.

[3] F. Calogero, Motion of poles and zeros of special solutions of nonlinear and linear partial differential equations, and related "solvable" many body problems, Nuovo Cimento 43B (1978) 177-241.

[4] F. Calogero, Classical many-body problems amenable to exact treatments (Lecture Notes in Physics Monographs m66, Springer, Heidelberg, 2001).

[5] F. Calogero, Isochronous systems (Oxford University Press, Oxford, 2008; marginally updated paperback edition 2012).

[6] D. Gómez-Ullate and M. Sommacal, Periods of the goldfish many-body problem, J. Nonlinear Math. Phys. 12, Suppl. 1 (2005) 351-362.

[7] F. Calogero, New solvable variants of the goldfish many-body problem, Studies Appl. Math., in press, available online; DOI: 10.1111/sapm.12096".

[8] A. Erdélyi (editor), Higher transcendental functions, vol. II (McGraw-Hill, New York, 1953).

[9] M. A. Olshanetsky and A. M. Perelomov, Classical integrable finite-dimensional systems related to Lie algebras, Phys. Rep. 71 (1981) 313-400. 